\title{
Application of tight-binding method to calculate the band structure and the effect of pressure in crystal $\mathrm{ZnSe}$
}

\author{
Hussein A. H. Sultan ${ }^{1 *}$, Mumtaz M. S. Hussien² \\ ${ }^{1 *, 2}$ Department of Physics, College of Education for pure science, University of Mosul, Iraq \\ E-mail: ${ }^{1 *}$ Hussein.esp127@student.uomosul.edu.iq, ${ }^{2}$ momtaz_hussien@uomosul.edu.iq
}

(Received February 02, 2021; Accepted March 23, 2021; Available online June 01, 2021)

DOI: 10.33899/edusj.2021.129479.1141, (c) 2021, College of Education for Pure Science, University of Mosul.

This is an open access article under the CC BY 4.0 license (http://creativecommons.org/licenses/by/4.0/).

\begin{abstract}
In this research tight-binding method has been applied to calculate the band structure in ZnSe crystal, the matrix elements of have been calculated using the method used by Vogl and Cohen. A computer program has been designed in MATLAB language to calculate the band structure in the ZnSe crystal, a sample of points has been formed in the first Brillouin zone (reduced zone) between the high symmetry points $(L \rightarrow \Gamma, \Gamma \rightarrow X \rightarrow(U, K) \rightarrow \Gamma)$. The energy eigen values is calculated along the high symmetry paths, the obtained results have been compared with previous works of Vogl and Cohen which shows a good agreements. A comparison between the $\mathrm{ZnSe}$ band structure based on $\mathrm{sp}^{3}$ and $\mathrm{sp}^{3} \mathrm{~s}^{*}$ models has been done and the energy gap between the conduction and valence bands at the high symmetry points is calculated for the $\mathrm{sp}^{3} \mathrm{~s}^{*}$. The effect of pressure on the ZnSe band structure is calculated in the range (10-40) Gpa by calculating the matrix element under different pressure, the results show broadening in band gap due to applied pressure, the conduction band is shifted toward the high energy while the valence band is shifted toward the lower energy. The band gap is calculated values for high symmetric points were determined with pressure change and compared with theoretical calculations.
\end{abstract}

Keywords: band structure, tight-binding method, effect of pressure on $\mathrm{ZnSe}$

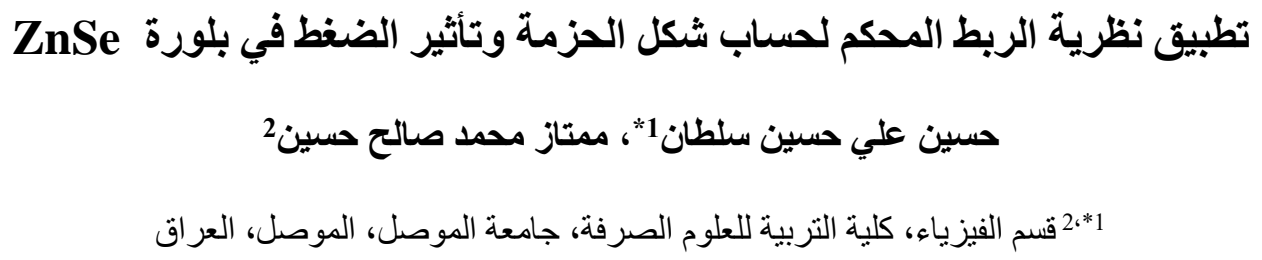

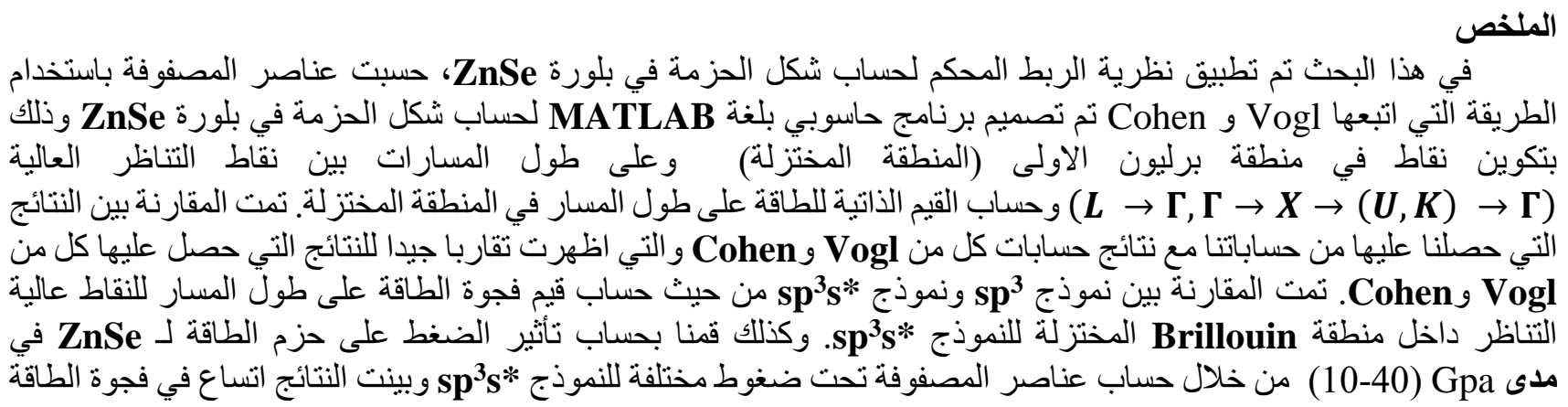


وزحزحة للحزم نحو الاعلى في حزمة التوصيل وزحزحة نحو الاسفل في حزمة التكافؤ مع زيادة الضغط. تم تحديد قيم فجوة الطاقة Egap

ZnSe الكلمات الدالة : نظرية الربط المحكم، نركيب الحزمة، تأثير الضغط على بلورة

المقدمة

تكمن اهمية نظريات حزم الطاقة للمواد الصلبة البلورية في ان العديد من الخصائص البصرية و الفيزيائية المهمة للمو اد الصلبة

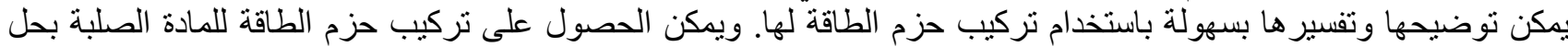

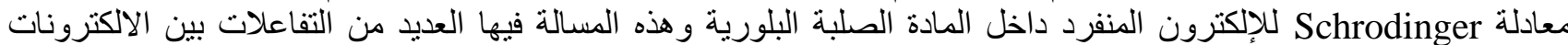

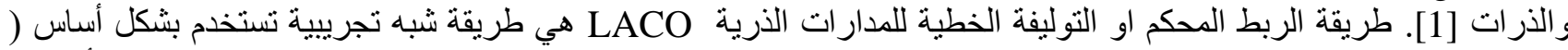

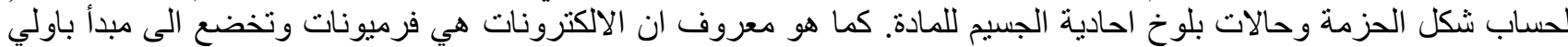

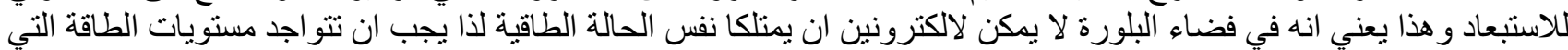

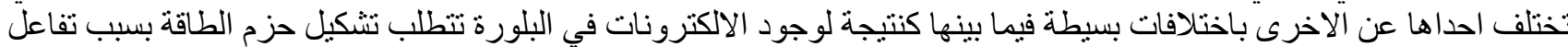

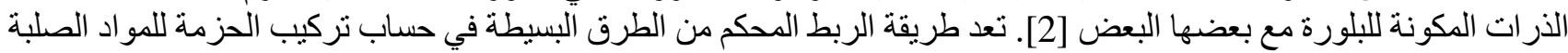

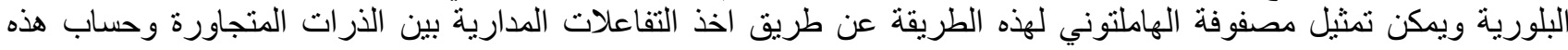

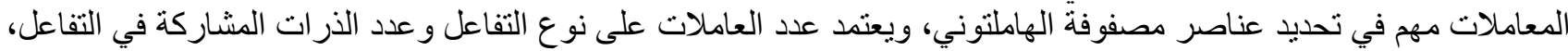

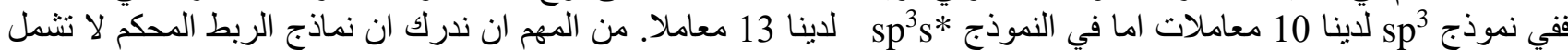

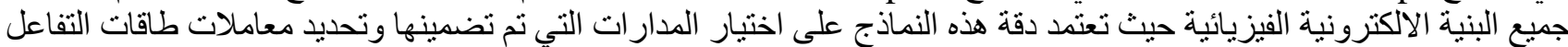

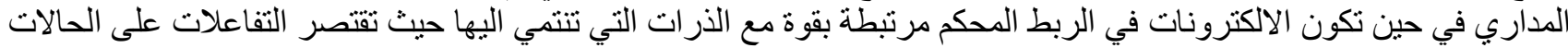

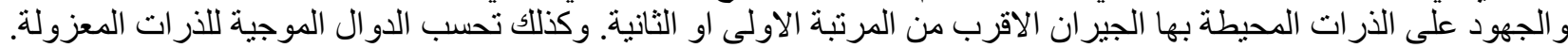

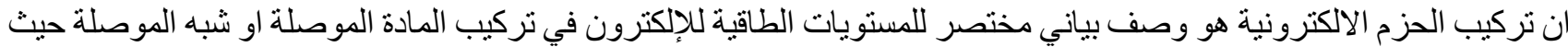

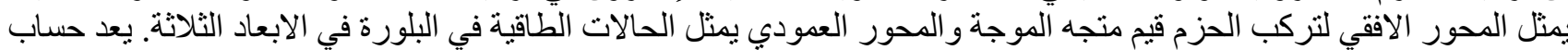

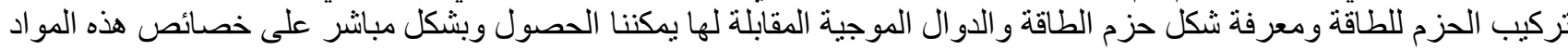

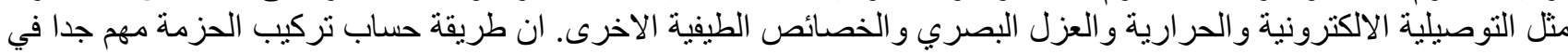

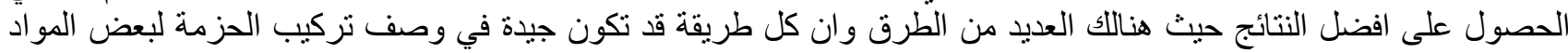

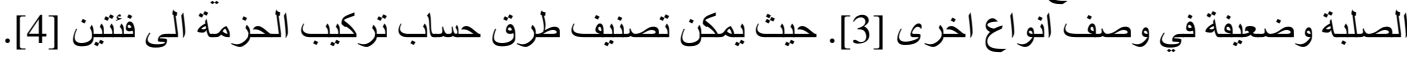

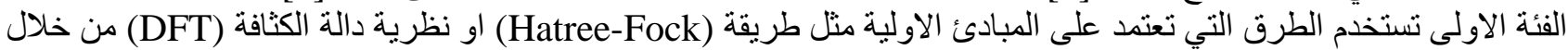

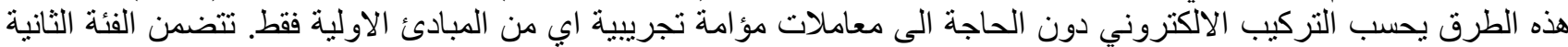

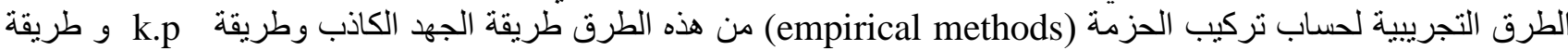

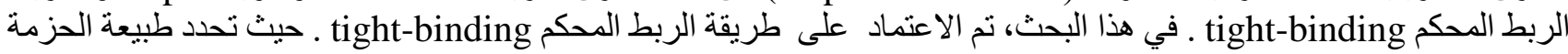

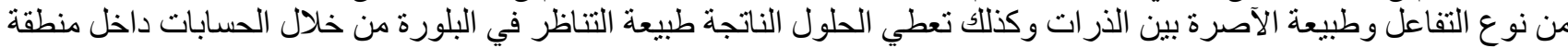

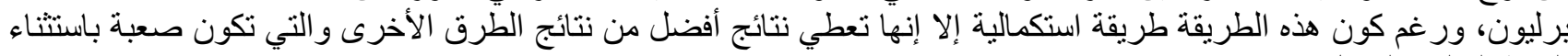

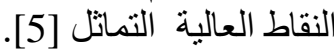

\section{الجزء النظري}

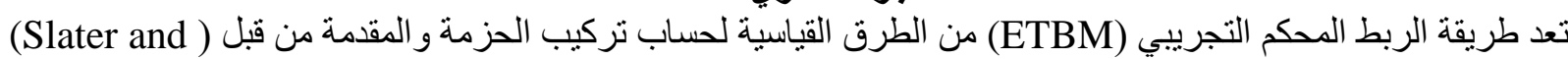

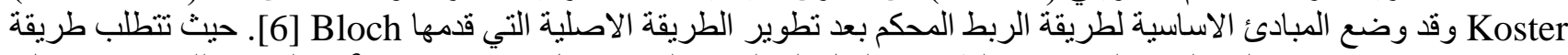

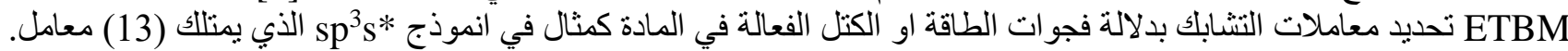

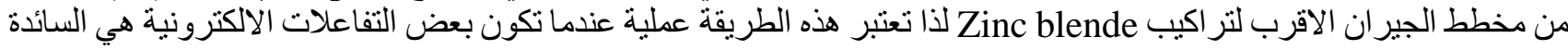

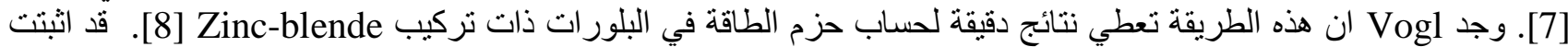

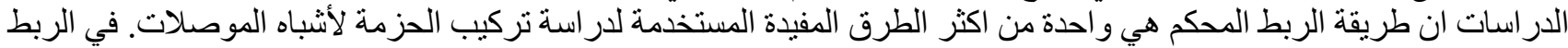

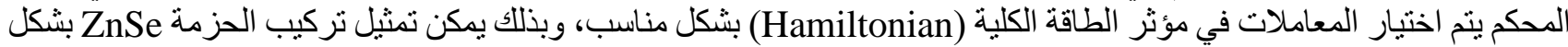

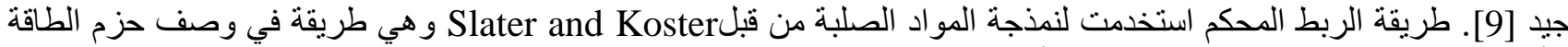

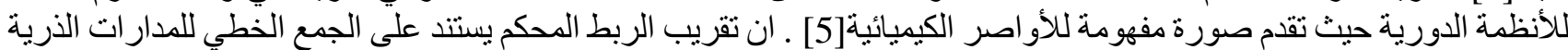

$$
\begin{aligned}
& H\left|\psi_{j}(\vec{r})\right\rangle=\varepsilon_{j}\left|\psi_{j}(\vec{r})\right\rangle \quad, j=1 \ldots \ldots \ldots N_{\text {tot }} \\
& H=\frac{P^{2}}{2 m_{e}}+V(\vec{r})
\end{aligned}
$$

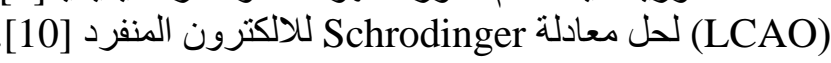


إذ ان H هو الهاملتونين، V(r) تمثل الجهد ويتضمن التفاعل الناتج من جميع الالكترونات الاخرى في النظام اضافة الى النوى في النظام البلوري. يمتلك الجهذ صنفة الدورية للثبيكة

$H(\vec{r}+\vec{R})=H(\vec{r})$ مؤثر الطاقة يصبح دوريا أيضا حيث

$\psi_{k j}(\vec{r}+\vec{R})=e^{i \vec{k} \cdot \vec{R}} \psi_{j}(\vec{r})$ و الدوال الذاتية للإلكترون المنفرد تحقق نظرية بلوخ

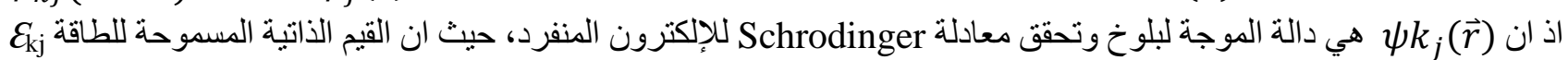
تتغير في كل مستوي بانسجام مع متجه الموجة k ونتشكل تركيب الحزمة للبلورة، دالة الموجة للإلكترون المنفرد يمكن ان يمثل كتر اكب المبل

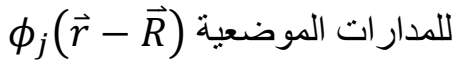

$\psi_{k j}(\vec{r})=\frac{1}{\sqrt{N}} \sum e^{i \vec{k} \cdot \vec{R}} \phi_{j}(\vec{r}-\vec{R})$

$\phi_{j}(\vec{r})=\sum_{n} C_{n j} C_{n}(\vec{r}) \quad$ تمثل عدد مو اقع الثبيكة حيث يمكن ان تأخذ المدارات الموضعية كجمع خطي للمدارات الذرية مثل الدوال $\psi_{k j}(\vec{r})=\sum_{n} C_{n j}(k) \mathrm{b}_{k n}(\vec{r})$

$\mathrm{b}_{k n}(\vec{r})=\frac{1}{\sqrt{N}} \sum_{R} e^{i \vec{k} \cdot \vec{R}} \varphi_{j}(\vec{r}-\vec{R})$

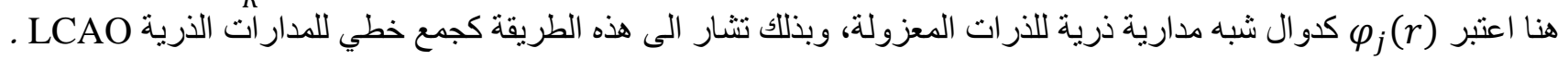
$\mathrm{H}_{\mathrm{nm}}=\left\langle\mathrm{b}_{\mathrm{n}}|\mathrm{H}| \mathrm{b}_{\mathrm{m}}\right\rangle=\frac{1}{\mathrm{~N}} \sum_{\mathrm{R} . \mathrm{R}^{\prime}} \mathrm{e}^{-\mathrm{ik} \cdot\left(\overrightarrow{\mathrm{R}}-\overrightarrow{\mathrm{R}^{\prime}}\right)} \int \varphi_{\mathrm{m}}^{*}(\overrightarrow{\mathrm{r}}-\overrightarrow{\mathrm{R}}) \cdot \mathrm{H} \varphi_{\mathrm{n}}(\overrightarrow{\mathrm{r}}-\overrightarrow{\mathrm{R}}) \mathrm{dr}$ $=\sum_{\mathrm{R}} \mathrm{e}^{-\mathrm{i} \overrightarrow{\mathrm{k}} \cdot \overrightarrow{\mathrm{R}}} \int \varphi_{\mathrm{m}}^{*}(\overrightarrow{\mathrm{r}}) \mathrm{H} \varphi_{\mathrm{n}}(\overrightarrow{\mathrm{r}}-\overrightarrow{\mathrm{R}}) \mathrm{dr}$

$S_{n m}=\left\langle b_{n} \mid b_{m}\right\rangle=\sum_{R} e^{-i \vec{k} \cdot \vec{R}} \int \varphi_{m}^{*}(\vec{r}) \cdot \varphi_{n}(\vec{r}-\vec{R}) d r$ وبشكل مشابه فان مصفوفة التشابك

$\sum \mathrm{H}_{\mathrm{nm}}(\mathrm{k}) \mathrm{C}_{\mathrm{mj}}(\mathrm{k})=\varepsilon_{\mathrm{kj}} \sum \mathrm{S}_{\mathrm{nm}}(\mathrm{k}) \mathrm{C}_{\mathrm{mj}}(\mathrm{k})$ حيث تصبح معادلة القيمة الذاتية المميزة $\left(\mathrm{H}(\mathrm{k})-\varepsilon_{k j} \cdot S(k)\right) C_{j}=0$

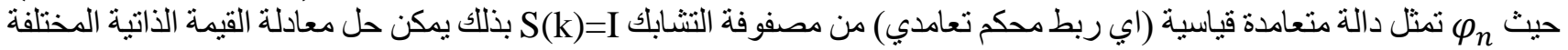

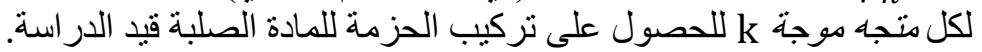

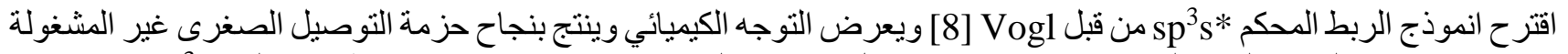

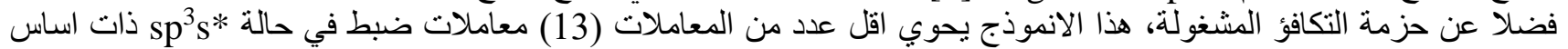

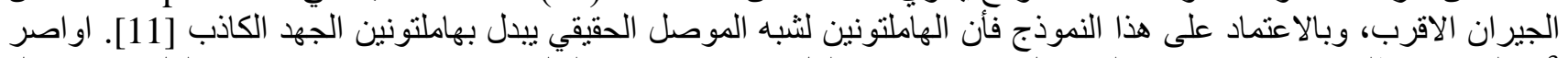

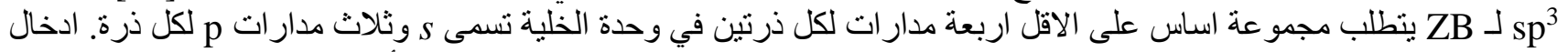

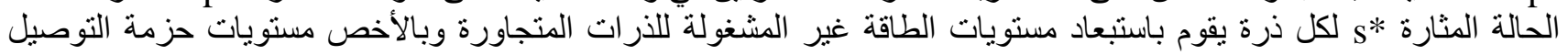

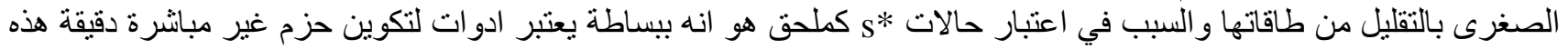

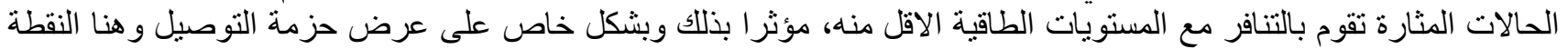

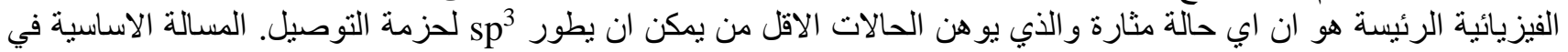

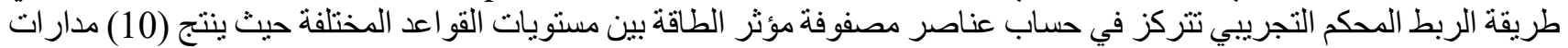

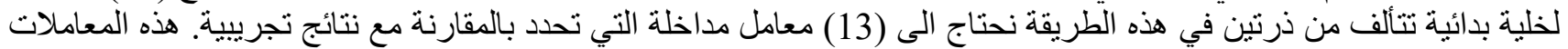

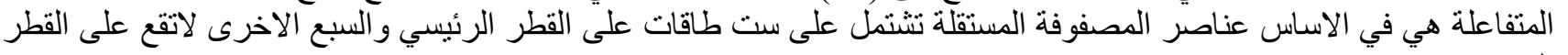




$$
\left.\begin{array}{l}
E(s, b)=\langle\mathrm{sb} \mathbf{R}|\mathrm{H}| \mathrm{sb} \mathbf{R}\rangle \\
E(p, b)=\left\langle\mathrm{p}_{\mathrm{x}} \mathrm{b} \mathbf{R}|\mathrm{H}| \mathrm{p}_{\mathrm{x}} \mathrm{b} \mathbf{R}\right\rangle \\
V(s, s)=4\langle\mathrm{saR}|\mathrm{H}| \mathrm{scR}\rangle \\
V(x, x)=4\left\langle\mathrm{p}_{\mathrm{x}} \mathrm{aR}|\mathrm{H}| \mathrm{p}_{\mathrm{x}} c \mathbf{R}\right\rangle \\
V(x, y)=4\left\langle\mathrm{p}_{\mathrm{x}} \mathrm{a} \mathbf{R}|\mathrm{H}| \mathrm{p}_{\mathrm{y}} c \mathbf{R}\right\rangle \\
V(s a, p c)=4\left\langle\mathrm{saR}|\mathrm{H}| \mathrm{p}_{\mathrm{x}} c \mathbf{R}\right\rangle \\
V(p a, s c)=4\left\langle\mathrm{p}_{\mathrm{x}} \mathrm{aR}|\mathrm{H}| \mathrm{scR}\right\rangle \\
E\left(s^{*}, b\right)=\left\langle\mathrm{s}^{*} \mathrm{~b} \mathbf{R}|\mathrm{H}| \mathrm{s}^{*} \mathrm{bR}\right\rangle \\
V\left(s^{*} a, p c\right)=4\left\langle\mathrm{~s}^{*} \mathrm{a}|\mathrm{R}| \mathrm{p}_{\mathrm{x}} \mathbf{R}\right) \\
V\left(p a, s^{*} c\right)=4\left\langle\mathrm{p}_{\mathrm{x}} \mathrm{aR}|\mathrm{H}| \mathrm{s}^{*} c \mathbf{R}\right\rangle
\end{array}\right\}
$$

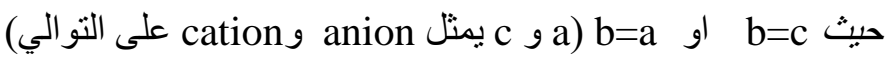

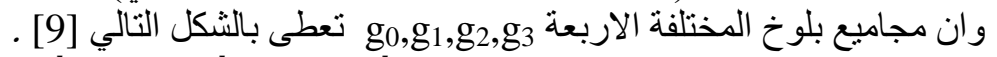

$g 0(k)=\cos \left(\frac{k_{1} a_{L}}{4}\right) \cos \left(\frac{k_{2} a_{L}}{4}\right) \cos \left(\frac{k_{3} a_{L}}{4}\right)-\mathrm{i} \sin \left(\frac{k_{1} a_{L}}{4}\right) \sin \left(\frac{k_{2} a_{L}}{4}\right) \sin \left(\frac{k_{3} a_{L}}{4}\right)$

$\mathrm{g} 1(\mathrm{k})=-\cos \left(\frac{k_{1} a_{L}}{4}\right) \sin \left(\frac{k_{2} a_{L}}{4}\right) \sin \left(\frac{k_{3} a_{L}}{4}\right)+\mathrm{i} \sin \left(\frac{k_{1} a_{L}}{4}\right) \cos \left(\frac{k_{2} a_{L}}{4}\right) \cos \left(\frac{k_{3} a_{L}}{4}\right)$

$\mathrm{g} 2(\mathrm{k})=-\sin \left(\frac{k_{1} a_{L}}{4}\right) \cos \left(\frac{k_{2} a_{L}}{4}\right) \sin \left(\frac{k_{3} a_{L}}{4}\right)+\mathrm{i} \cos \left(\frac{k_{1} a_{L}}{4}\right) \sin \left(\frac{k_{2} a_{L}}{4}\right) \cos \left(\frac{k_{3} a_{L}}{4}\right)$

$\mathrm{g} 3(\mathrm{k})=-\sin \left(\frac{k_{1} a_{L}}{4}\right) \sin \left(\frac{k_{2} a_{L}}{4}\right) \cos \left(\frac{k_{3} a_{L}}{4}\right)+\mathrm{i} \cos \left(\frac{k_{1} a_{L}}{4}\right) \cos \left(\frac{k_{2} a_{L}}{4}\right) \sin \left(\frac{k_{3} a_{L}}{4}\right)$

$\mathrm{k}=\left(\frac{2 \pi}{a}\right)\left(k_{1}, k_{2}, k_{3}\right)$

$$
\text { هنا متجه الموجة يعطى بدلالة المركبات الثلاثة (1) }
$$

\section{الحسابات والنتائج}

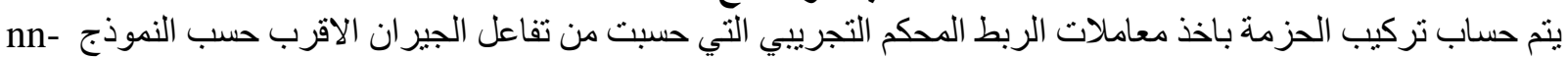

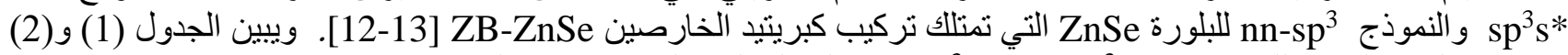

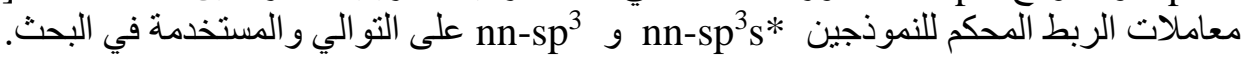

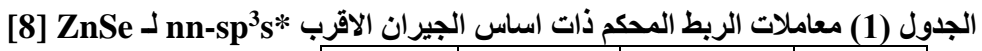

\begin{tabular}{|l|l|l|l|}
\hline $\mathrm{E}(\mathrm{s}, \mathrm{a})$ & -11.8383 & $\mathrm{~V}(\mathrm{x}, \mathrm{x})$ & 3.0054 \\
\cline { 1 - 2 } $\mathrm{E}(\mathrm{p}, \mathrm{a})$ & 1.5072 & $\mathrm{~V}(\mathrm{x}, \mathrm{y})$ & 5.9942 \\
$\mathrm{E}(\mathrm{s}, \mathrm{c})$ & 0.0183 & $\mathrm{~V}(\mathrm{sa}, \mathrm{pc})$ & 3.4980 \\
\hline $\mathrm{E}(\mathrm{p}, \mathrm{c})$ & 5.9928 & $\mathrm{~V}(\mathrm{sc}, \mathrm{pa})$ & 6.3191 \\
\cline { 1 - 2 } $\mathrm{E}\left(\mathrm{s}^{*}, \mathrm{a}\right)$ & 7.5872 & $\mathrm{~V}\left(\mathrm{~s}^{*} \mathrm{a}, \mathrm{pc}\right)$ & 2.5891 \\
\cline { 1 - 2 } $\mathrm{E}\left(\mathrm{s}^{*}, \mathrm{c}\right)$ & 8.9928 & $\mathrm{~V}\left(\mathrm{pa}, \mathrm{s}^{*} \mathrm{c}\right)$ & 3.9533 \\
\cline { 1 - 2 } $\mathrm{V}(\mathrm{s}, \mathrm{s})$ & -6.2163 & & \\
\hline
\end{tabular}

[13] ZnSe لـ Sp3 الجدول (2) معاملات الربط المحكم ذات اساس الجيران الاقرب

\begin{tabular}{|l|l|l|l|}
\hline $\mathrm{Es}_{0}$ & -8.92 & $\mathrm{Vs}_{0} \mathrm{p}$ & 5.47 \\
\hline $\mathrm{Es}_{1}$ & -0.28 & $\mathrm{Vs}_{1} \mathrm{p}$ & 4.73 \\
\hline $\mathrm{Ep}_{0}$ & 0.12 & $\mathrm{Vxy}$ & 4.38 \\
\hline $\mathrm{Ep}_{1}$ & 7.42 & $\mathrm{Vxx}$ & 0.96 \\
\hline $\mathrm{Vss}$ & -6.14 & & \\
\hline
\end{tabular}


بعد ايجاد عناصر المصفوفة يتم حساب المحدد العام (secular determinant) لكل التفاعلات الممكنة. حيث يمكن تقليل مدى در اسة

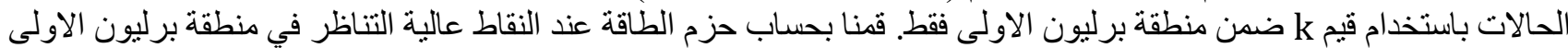

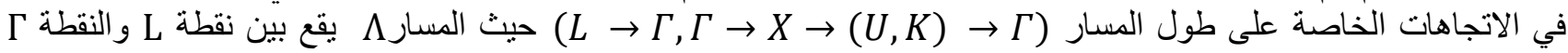

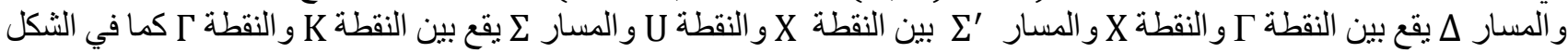

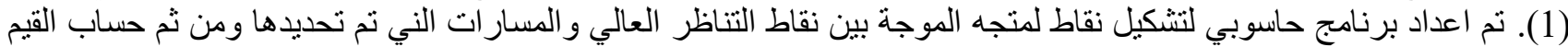

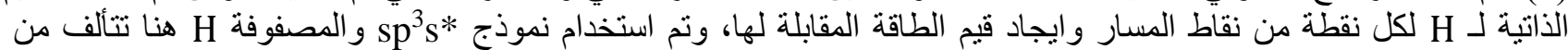

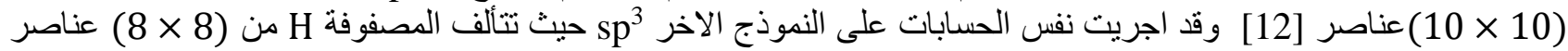

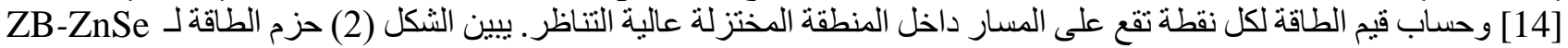

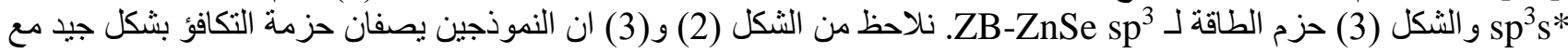

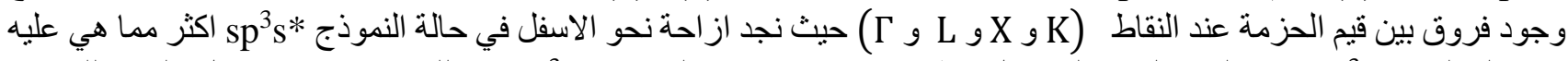

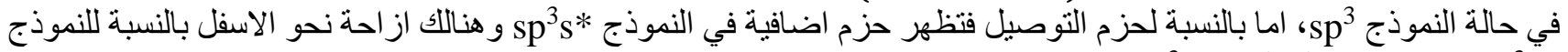

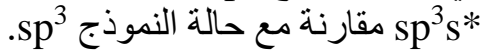

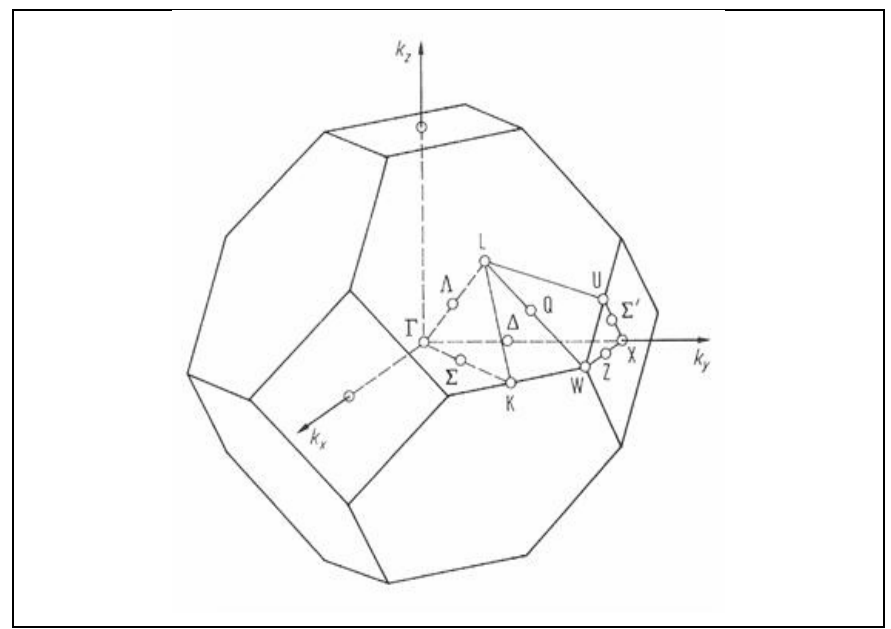

Fcc الثنكل (1) منطقة برليون الاولى والمنطقة المختزلة لـ F F

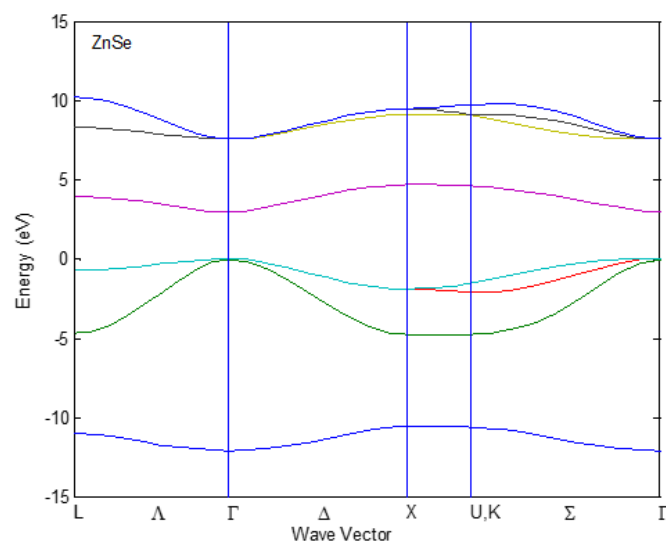

Sp

الثكل (3) :

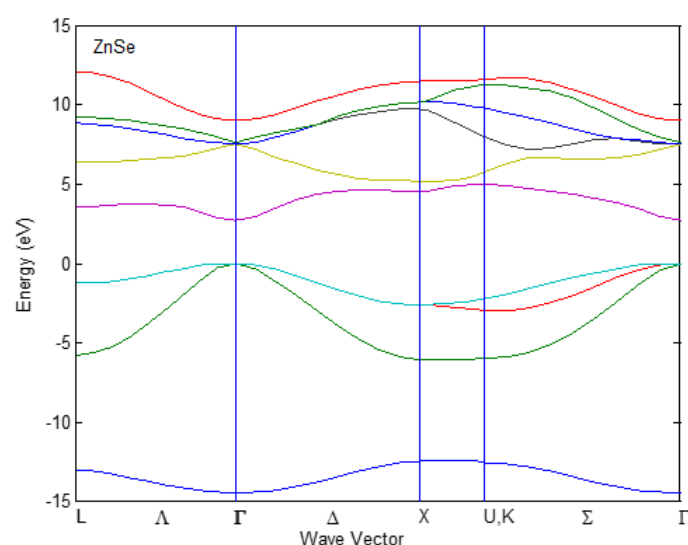

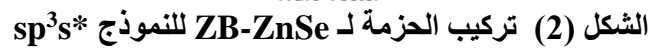

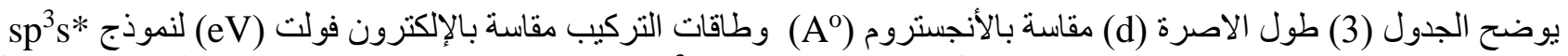

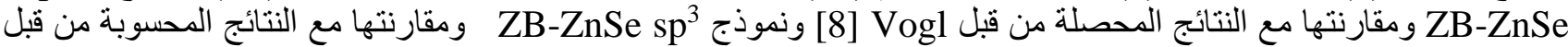
Cohen [13 [يث وجدنا نطابق كبير بين النتائج التي حصلنا عليها في هذا البحث وبين النتائج المحصلة من قبل 


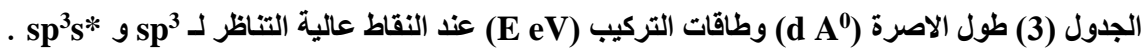

\begin{tabular}{|c|c|c|c|c|}
\hline ZnSe & Vogl & Present work & Cohen & Present work \\
\hline $\mathrm{d}$ & 2.45 & 2.45 & 2.45 & 2.45 \\
\hline $\boldsymbol{\Gamma}_{\mathbf{1}}^{\boldsymbol{v}}$ & -14.50 & -14.4999 & -12.1 & -12.1075 \\
\hline $\boldsymbol{\Gamma}_{\mathbf{1}}^{\boldsymbol{C}}$ & 2.68 & 2.6799 & 2.9 & 2.9075 \\
\hline $\boldsymbol{\Gamma}_{\mathbf{1 5}}^{\boldsymbol{C}}$ & 7.50 & 7.5000 & 7.5 & 7.5441 \\
\hline $\mathbf{X}_{\mathbf{1}}^{\boldsymbol{v}}$ & -12.50 & -12.5117 & -10.6 & -10.5821 \\
\hline $\mathbf{X}_{\mathbf{3}}^{\boldsymbol{v}}$ & -5.60 & -6.0789 & -4.8 & -4.8142 \\
\hline $\mathbf{X}_{\mathbf{5}}^{\boldsymbol{v}}$ & -2.65 & -2.6500 & -1.9 & -1.9315 \\
\hline $\mathbf{X}_{\mathbf{1}}^{\boldsymbol{C}}$ & 4.5400 & 4.5400 & 4.7 & 4.6542 \\
\hline $\mathbf{X}_{\mathbf{3}}^{\boldsymbol{C}}$ & 5.1700 & 5.1700 & 9.0 & 9.0821 \\
\hline
\end{tabular}

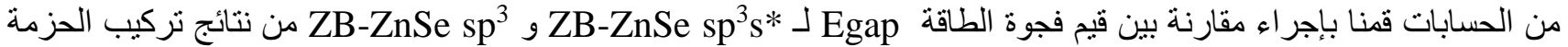
و التي حصلنا عليها باستخدام البرنامج الحاسوبي الذي تم اعداء التياده في الدراسة، ويبين الجدول (4) قيم فجوة الطاقة المبانشرة وغير

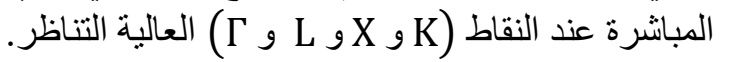

الجدول (4) المقارنة بين قيم Egap(eV) عند نقاط التناظر ــ sp3 و sp3s*

\begin{tabular}{|c|c|c|}
\hline $\operatorname{Egap}(\mathrm{eV})$ & $\mathrm{Sp}^{3}$ & $\mathrm{Sp}^{3} \mathrm{~s}^{*}$ \\
\hline $\operatorname{Egap}(\Gamma)$ & 2.9075 & 2.6799 \\
\hline $\operatorname{Egap}(\mathrm{L})$ & 3.8808 & 3.5788 \\
\hline $\operatorname{Egap}(\mathrm{X})$ & 4.6542 & 4.5400 \\
\hline $\operatorname{Egap}(\mathrm{K})$ & 4.5868 & 4.9204 \\
\hline
\end{tabular}

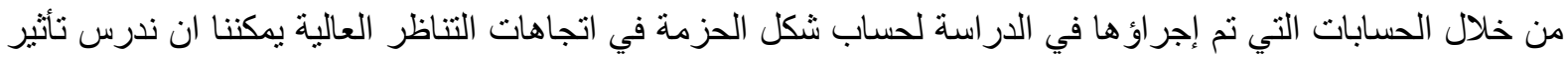

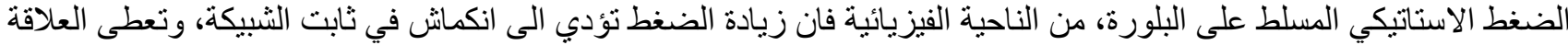
بين ثابت الثبيكة عند تسليط الضغط بدلالة عامل المرونة الحجمي بالثكل التالي [15 [1:

$$
a_{P}=a_{o}\left(1+\frac{P B^{\prime}}{B_{S}}\right)^{\frac{-1}{3 B^{\prime}}}
$$

حيث $a_{P}$

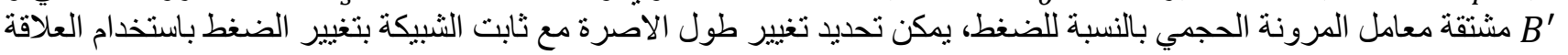

$$
d_{P}=d_{o}\left(\frac{a_{o}}{a_{p}}\right)
$$

الاتية:

de $d_{P}$

$$
V_{\alpha \beta}=V_{\alpha \beta}(0)\left(\frac{d_{p}}{d_{o}}\right)^{n(\alpha \beta)}
$$

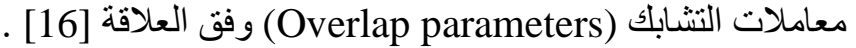

حيث ان لمر و و (0)

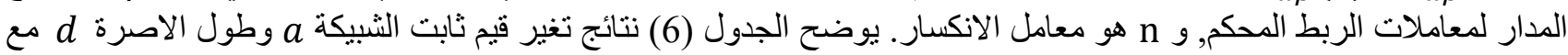

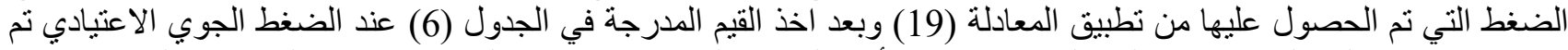

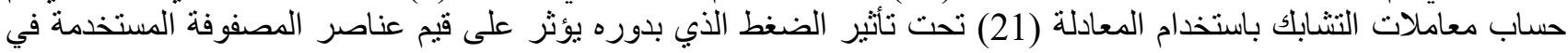

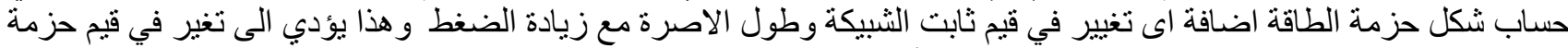

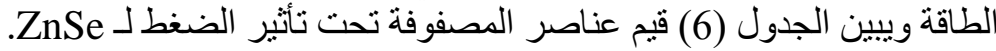


a. Ref,[17]

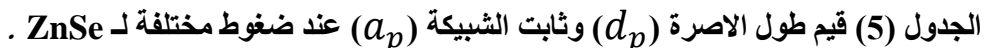

\begin{tabular}{|c|c|c|}
\hline $\mathrm{P}(\mathrm{Gpa})$ & $a_{p}\left(\mathrm{~A}^{0}\right)$ & $d_{p}\left(\mathrm{~A}^{0}\right)$ \\
\hline 0 & $5.6692^{(\mathrm{a})}$ & $2.4542^{(\mathrm{a})}$ \\
\hline 5 & 5.4877 & 2.3762 \\
\hline 10 & 5.3838 & 2.3312 \\
\hline 15 & 5.2982 & 2.2942 \\
\hline 20 & 5.2229 & 2.2616 \\
\hline 25 & 5.1547 & 2.2320 \\
\hline 30 & 5.0917 & 2.2047 \\
\hline 35 & 5.0328 & 2.1793 \\
\hline 40 & 4.9774 & 2.1552 \\
\hline
\end{tabular}

\begin{tabular}{|c|c|c|c|c|c|}
\hline \multicolumn{6}{|c|}{ الجدول (6) قيم عناصر المصفوفة عند ضغوط مختلفة. } \\
\hline \multirow{2}{*}{$\begin{array}{c}\text { Matrix } \\
\text { elements }\end{array}$} & \multicolumn{5}{|c|}{ Pressure $\mathrm{p}(\mathrm{Gpa})$} \\
\hline & 0 & 10 & 20 & 30 & 40 \\
\hline Esa & -11.8383 & -13.4025 & -14.4160 & -15.3250 & $16.1846-$ \\
\hline Epa & 1.5072 & 1.7064 & 1.8354 & 1.9511 & 2.0606 \\
\hline Esc & 0.0183 & 0.0207 & 0.0223 & 0.0237 & 0.0250 \\
\hline Epc & 5.9928 & 6.7846 & 7.2977 & 7.7578 & 8.1930 \\
\hline Es*a & 7.5872 & 8.5897 & 9.2393 & 9.8218 & 10.3728 \\
\hline Es*c & 8.9928 & 10.1810 & 10.9509 & 11.6414 & 12.2944 \\
\hline Vss & -6.2163 & -7.0377 & -7.5699 & -8.0472 & $8.4985-$ \\
\hline Vxx & 3.0054 & 3.4025 & 3.6598 & 3.8906 & 4.1088 \\
\hline Vxy & 5.9942 & 6.7862 & 7.2994 & 7.7596 & 8.1949 \\
\hline Vsp & 3.4980 & 3.9602 & 4.2597 & 4.5283 & 4.7823 \\
\hline Vps & 6.3191 & 7.1541 & 7.6951 & 8.1802 & 8.6391 \\
\hline Vs*p & 2.5891 & 2.9312 & 3.1529 & 3.3517 & 3.5397 \\
\hline Vps* & 3.9533 & 4.4757 & 4.8141 & 5.1177 & 5.4047 \\
\hline
\end{tabular}

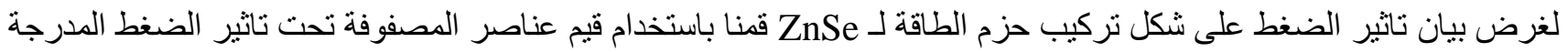

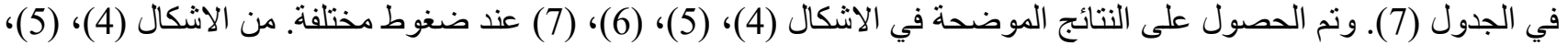

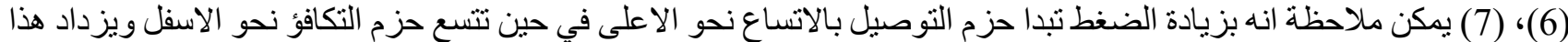

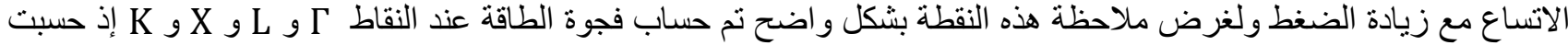

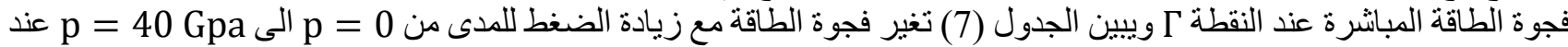

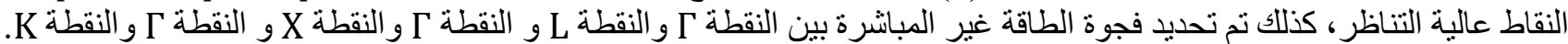

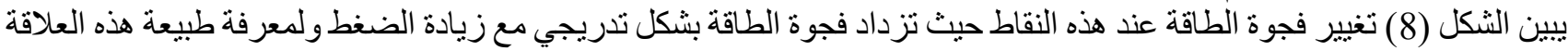

$$
\mathrm{E}_{\mathrm{g}}(\mathrm{p})=\gamma+\beta \mathrm{p}+\alpha \mathrm{p}^{2}
$$

لفجوة الطاقة مع الضغط و الني تكون بشكل علاقة من الدرجة الثنانية

اذ ان م و م و و هي معاملات يمكن حسابها عن طريق موائمة النتائج التي تم الحصول عليها من حسابات تركيب الحزمة عند

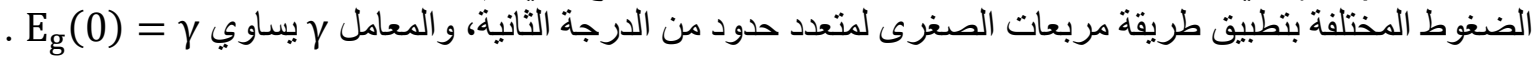




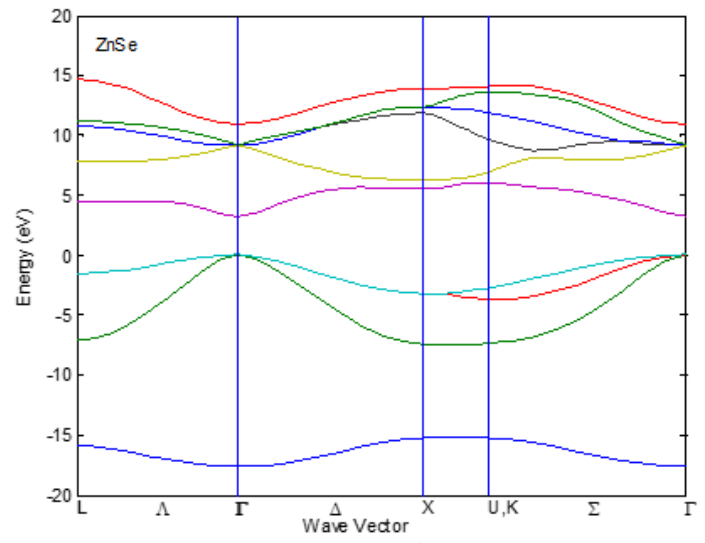

الشكل (5) : تركيب الحزمة لـ ZnSe Gpa) عند الضغط

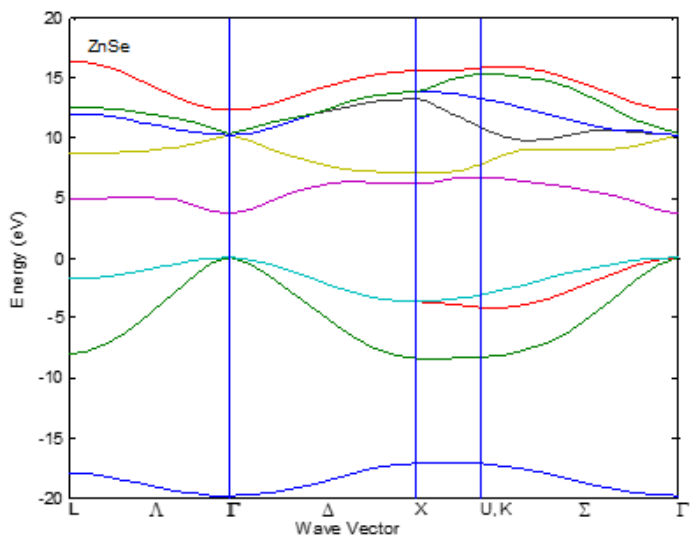

(40 Gpa) الشكل (7) : تركيب الحزمة لـ ZnSe عند الضغط

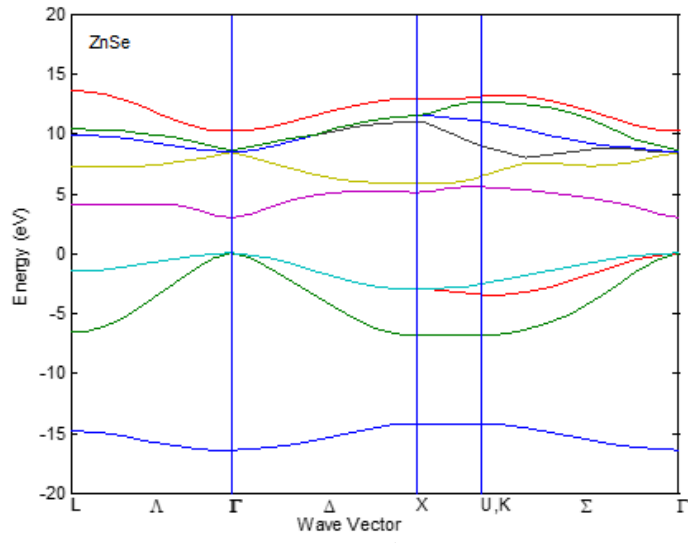

(10 Gpa) الثكل (4) : تركيب الحزمة لـ ZnSe عند الضغط

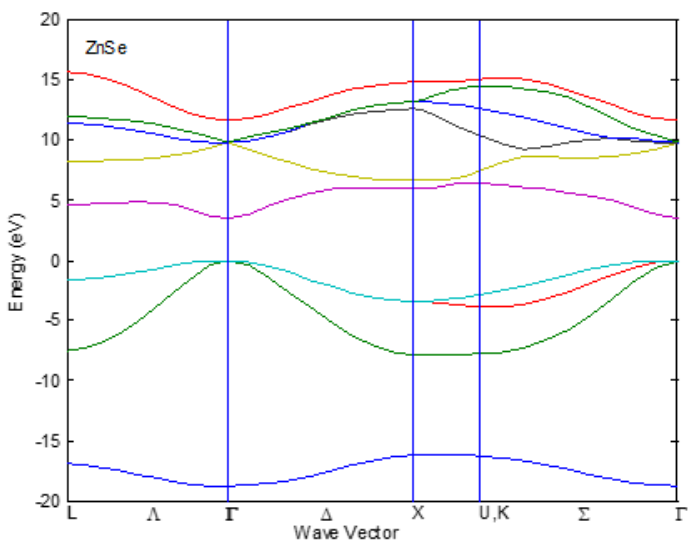

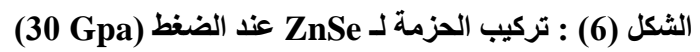

\begin{tabular}{|c|c|c|c|c|}
\hline $\mathrm{P}(\mathrm{Gpa})$ & $\operatorname{Eg}(\Gamma \Gamma)$ & $\operatorname{Eg}(\Gamma L)$ & $\operatorname{Eg}(\Gamma X)$ & $\operatorname{Eg}(\Gamma K)$ \\
\hline 0 & 2.6799 & 3.5788 & 4.5400 & 4.9204 \\
\hline 5 & 2.8979 & 3.8699 & 4.9092 & 5.3206 \\
\hline 10 & 3.0341 & 4.0517 & 5.1399 & 5.5705 \\
\hline 15 & 3.1531 & 4.2108 & 5.3417 & 5.7893 \\
\hline 20 & 3.2635 & 4.3771 & 5.5286 & 5.9918 \\
\hline 25 & 3.3683 & 4.4980 & 5.7061 & 6.1841 \\
\hline 30 & 3.4693 & 4.6329 & 5.8771 & 6.3695 \\
\hline 35 & 3.5676 & 4.7642 & 6.0437 & 6.5501 \\
\hline 40 & 3.6638 & 4.8927 & 6.2068 & 6.7269 \\
\hline
\end{tabular}

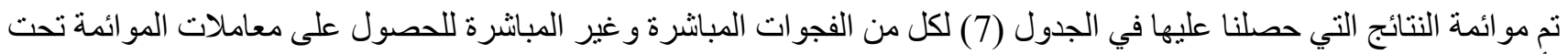

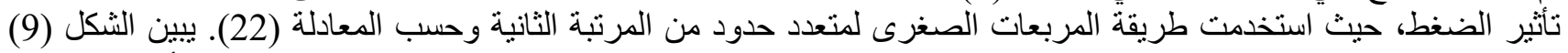

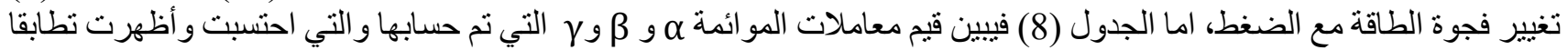
جيدا مع الحسابات التي قمنا بها باستخدام طريقة الربط المحكم التجريبية . 


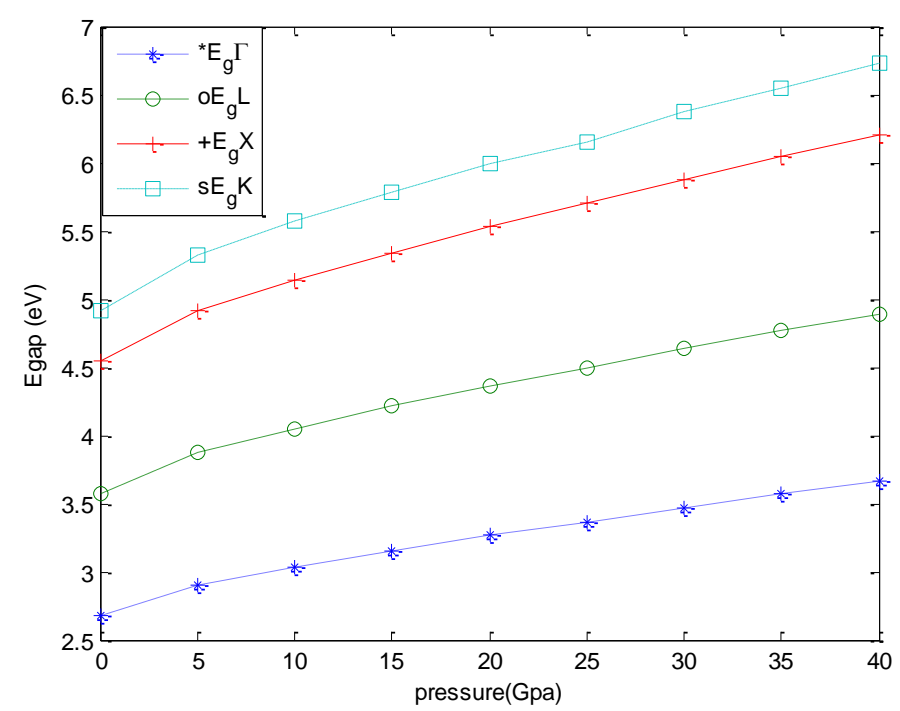

الثكل (8) تغيير فجوة الطاقة لبلورة ZnSe مع الضغط.

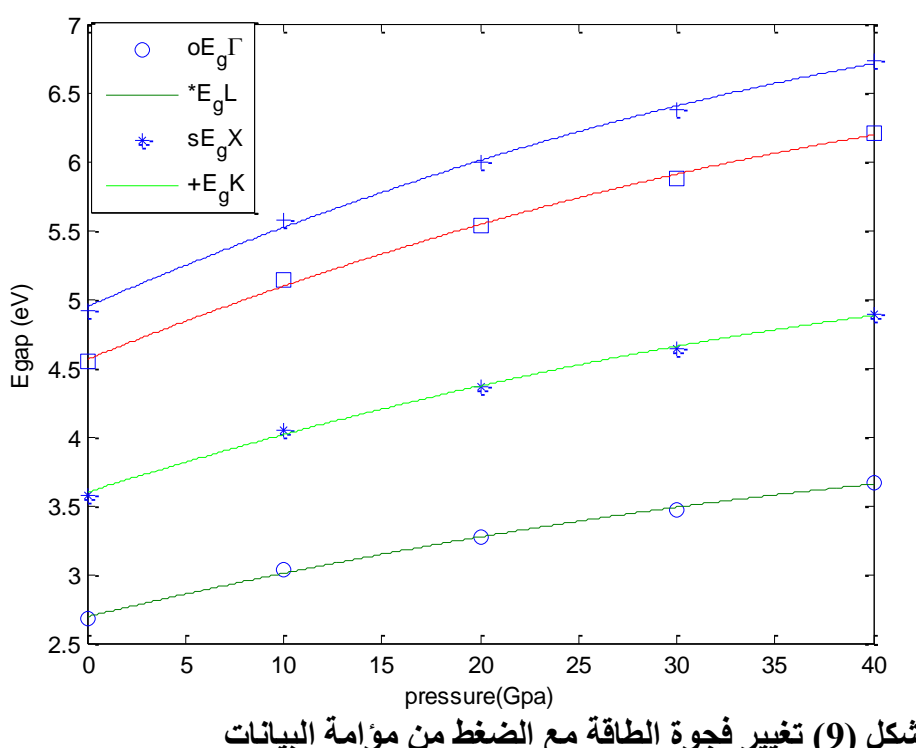

الجدول (8) حساب قير a و B و و عند نقاط النتاظز العالية

\begin{tabular}{|c|c|c|c|}
\hline Hight symmetry points & $\boldsymbol{\alpha}$ & $\boldsymbol{\beta}$ & $\gamma$ \\
\hline$\Gamma$ & $\mathbf{0 . 0 3 3 8}$ & $\mathbf{- 0 . 0 0 0 2}$ & $\mathbf{2 . 6 9 2 5}$ \\
\hline$L$ & $\mathbf{0 . 0 4 5 2}$ & $\mathbf{- 0 . 0 0 0 3}$ & $\mathbf{3 . 5 9 5 6}$ \\
\hline$X$ & $\mathbf{0 . 0 5 7 3}$ & $\mathbf{- 0 . 0 0 0 4}$ & $\mathbf{4 . 5 6 1 4}$ \\
\hline$K$ & $\mathbf{0 . 0 6 2 1}$ & $\mathbf{- 0 . 0 0 0 4}$ & $\mathbf{4 . 9 4 3 6}$ \\
\hline
\end{tabular}

\section{المناقثة}

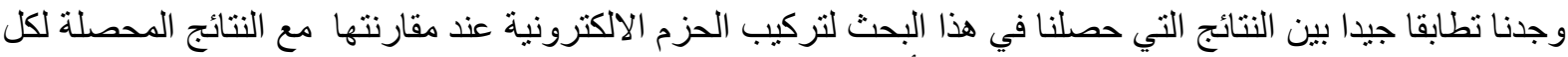

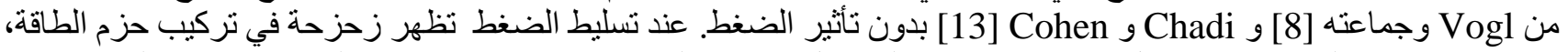

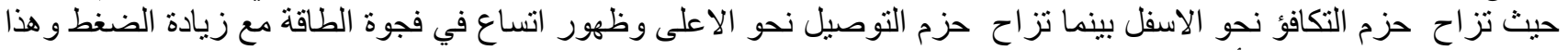

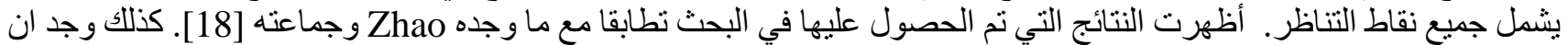

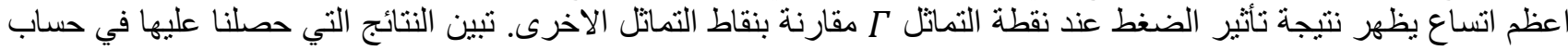
فجو ات الطاقة عند النقاط عالية التماثل تعطي تو افقا جيدا مع نتائج الابحاث المنشورة الاخرى [9] ، ؛ [13]. عند مقارنة قيم فجوة الطاقة 


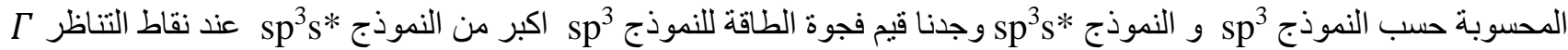

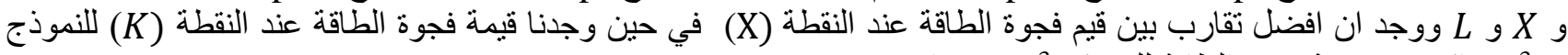

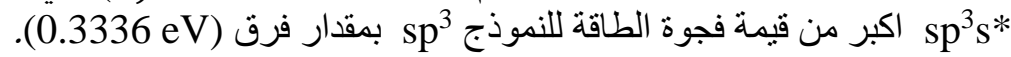
شكر و تقدير

يتقدم الباحثان بالثكر و التقدير الى عمادة كلية التربية للعلوم الصرفة وقسم الفيزياء على دعم البحث.

\section{المصادر}

[1]-Aschroft N.W.\& Mermin N.D.,"solid state physics" , Hoh Saunders:Tokyo (1981).

[2]-Zurich E., "Band Structure Effects and Quantum Transport.", Ph. D. dissertation (2010).

[3]- Gallaway, J. " Quantum theory of solid state" part A, Academic press, INC. New York (1974).

[4]- Yu P. Y.and Cardona;"Fundamentals of semiconductors"; Springer Verlag, Berlin:1-775 (1999)

[5]-Slater J. C. and Koster G. F., Phys. Rev.,94, 6:1498-1524 (1954).

[6] Bloch F., Z. Phys., 52, :555-600, (1928).

[7]- Vurgaftman, I., Meyer, J.R. J. Appl. Phys. 89, 11: 5815-5875 (2001).

[8]- Vogl, P.; Hjalmarson, H.P.; Dow, J.D. J. Phys. Chem. Solids, 44, 5: 365-378 (1983).

[9]- Hang, L.K.; Chun, H.M.; Zhong, Z.Z.; Peng, Z.Z. Chin. Phys. Lett. 16, 6: 437-439 (1999).

[10]- Leu, P.; Cai, W.,(pdf), ME 364. Stanford University:1-16 (2004).

[11] Austin B. J.. Heine V. and Sham L. J.. Phys. Rev.127, 276-282 (1962).

[12]- Gopir, G.; Zulkifli, N.O.; Othman, A. P., Solid State Science and Technology 13, 1 \& 2, 234243 (2005).

[13]- Chadi D. J. and Cohen M. L.; Phys. Stat. Sol. (b); 68: 405-419 (1975).

[14] Salcedo-Reyes JC., Universitas Scientiarum. 13(2):198-207, (2008).

[15]- Adachi, S. Properties of group IV, III-V and II-VI semiconductors, Wiley, N.Y., Chap (2005).

[16]- Contreras-Solorio, D. A, Reyes Villagrana R.A. and Madrigal Melchor J., Revista Mexicana de Física 53.7: 132-135 (2007).

[17]- Adachi, S." Properties of semiconductor alloys: group-IV, III-V and II-VI semiconductors". Vol. 28. John Wiley \& Sons, (2009).

[18]- Zhao, C.Z., Wei, T., Sun, X.D., Wang, S.S. and Lu, K.Q., Physica B: Condensed Matter 494:7174 (2016). 